\title{
Progression Free Survival Prediction for Head and Neck Cancer using Deep Learning based on Clinical and PET- CT Imaging Data
}

\author{
Mohamed A. Naser ${ }^{10000-0003-1020-4966]}$, Kareem A. Wahid ${ }^{10000-0002-0503-0175]}$, Abdallah \\ S.R. Mohamed ${ }^{10000-0003-2064-7613]}$, Moamen Abobakr Abdelaal ${ }^{1[0000-0003-4476-2122]}$, Renjie \\ $\mathrm{He}^{1[0000-0001-9166-6286]}$, Cem Dede ${ }^{1[0000-0002-0543-9325]}$, Lis anne V. van Dijk ${ }^{1[0000-0002-9515-}$ \\ 5616], and Clifton D. Fuller ${ }^{10000-0002-5264-3994]}$ \\ ${ }^{1}$ Department of Radiation Oncology, The University of Texas MD Anderson Cancer, Houston, \\ Texas 77030, USA \\ manaser@mdanderson.org
}

\begin{abstract}
.
Determining progression-free survival (PFS) for head and neck squamous cell carcinoma (HNSCC) patients is a challenging but pertinent task that could help stratify patients for improved overall outcomes. PET/CT images provide a rich source of anatomical and metabolic data for potential clinical biomarkers that would inform treatment decisions and could help improve PFS. In this study, we participate in the 2021 HECKT OR Challenge to predict PFS in a large dataset of HNSCC PET/CT images using deep learning approaches. We develop a series of deep learning models based on the DenseNet architecture using a negative loglikelihood loss function that utilizes PET/CT images and clinical data as separate input channels to predict PFS in days. Internal model validation based on 10-fold cross-validation using the training data $(\mathrm{N}=224)$ yielded $\mathrm{C}$-index values up to 0.622 (without) and 0.842 (with) censoring stat us considered in C-index computation, respectively. We then implemented model ensembling approaches based on the training dat a cross-validation folds to predict the PFS of the test set patients $(\mathrm{N}=101)$. External validation on the test set for the best ensembling method yielded a C-index value of 0.694 . Our results are a promising example of how deep learning approaches can effectively utilize imaging and clinical data for medical outcome prediction in HNSCC, but further work in optimizing these processes is needed.
\end{abstract}

Keywords: PET, CT, Head and Neck Cancer, Oropharyngeal Cancer, Deep Learning, Progression-Free Survival, Outcome Prediction Model. 
medRxiv preprint doi: https://doi.org/10.1101/2021.10.14.21264955; this version posted October 17, 2021. The copyright holder for this preprint (which was not certified by peer review) is the author/funder, who has granted medRxiv a license to display the preprint in perpetuity.

It is made available under a CC-BY 4.0 International license.

\section{Introduction}

Head and neck squamous cell carcinomas (HNSCC) are among the most prevalent cancers in the world. Approximately 890,000 new cases of HNSCC are diagnosed a year, and rates are projected to continue to increase [1]. While prognostic outcomes for HNSCC, particularly for oropharyngeal cancer (OPC), have improved over recent years, patients still have a significant probability of disease recurrence or death [2]. Determination of progression-free survival (PFS) in HNSCC is a highly challenging task since the ultimate healthcare outcomes of patients are driven by a complex interaction between a large number of variables, including clinical demographics, treatment approaches, and underlying dis ease physiology. While risk prediction models based on clinical demographics for HNSCC have been developed in the past [3], these methods may lack prediction potential due to their use of a s mall number of simple variables or linear nature.

PET/CT imaging provides an avenue to combine anatomical with functional imaging to gauge the phenotypic properties of tumors. Dis tinct morphologic and metabolic information derived from PET/CT has been linked to the underlying pathology of HNSCC tumors and is invaluable in diagnosis, staging, and therapeutic as sessment [4]. Therefore, PET/CT imaging data has been as an attractive biomarker in developing clinical outcome prediction models for HNSCC. Radiomic analys sis of PET/CT images in HNSCC has been heavily investigated in the past, with models bas ed on statistical, geometric, and texture information contained within regions of interest showing promise in predicting PFS $[5,6]$. However, radiomic methods rely on hand-crafted features and pre-defined regions of interest, which can introduce bias into analysis pipelines [7]. Therefore, deep learning approaches, which do not require a-priori definition of regions of interest and where features are learned during the model training process, have been touted as attractive alternatives in the medical imaging domain [7-10]. However, while deep learning methods for clinical prediction are promis ing, they are often bottlenecked by small homogenous training and evaluation datasets that limit the generalizability of models [11]. Therefore, developing and validating deep learning models for HNSCC outcome prediction on large multi-institutional datasets is of paramount importance.

The 2021 HECKTOR Challenge provides an opportunity to utilize large heterogenous training and testing PET/CT datasets with matched clinical data to further unlock the power of clinical prediction models for HNSCC. This manuscript describes the development and evaluation of a deep learning prediction model based on the DenseNet architecture that can implement PET/CT images and clinical data to predict PFS for HNSCC patients. 
medRxiv preprint doi: https://doi.org/10.1101/2021.10.14.21264955; this version posted October $17,2021$. The copyright holder for this preprint (which was not certified by peer review) is the author/funder, who has granted medRxiv a license to display the preprint in

It is made available under a CC-BY 4.0 International license.

\section{Methods}

We developed and trained a deep learning model (2.3) through a cros s validation procedure (2.4) for predicting the progression-free survival (PFS) endpoint of OPC patients (based on censoring status and time-to-event between PET/CT scan and event) using co-registered ${ }^{18}$ F-FDG PET and CT imaging data (2.1) and as sociated clinical data (2.2). The performance of the trained model for predicting PFS was validated using a internal cros s-validation approach and applied to a previously unseen testing set (2.5).

\subsection{Imaging Data}

The data set used in this manuscript consists of co-registered ${ }^{18} \mathrm{~F}-\mathrm{FDGPET}$ and CT scans for 325 OPC patients (224 patients used for training and 101 patients used for testing). All training and testing data were provided in Neuroimaging Informatics Technology Initiative (NIfTI) format and were released through AIcrowd [12] for the HECKTOR Challenge at MICCAI 2021 [13].

All images (i.e., PET and CT) were cropped to fixed bounding box volumes, provided with the imaging databy [12], of size 144x144x144 $\mathrm{mm}^{3}$ in the $\mathrm{x}$, $\mathrm{y}$ and zdimensions and then resampled to a fixed image resolution of $1 \mathrm{~mm}$ in the $\mathrm{x}, \mathrm{y}$, and $\mathrm{z}$ dimensions using spline interpolation of order 3 . The cropping and resampling codes used were based on the code provided by the HECKTOR Challenge organizers (https://github.com/voreille/hecktor). The CT intensities were truncated in the range of $[-200,200]$ Hounsfield Units (HU) to increase soft tis sue contrast and then were normalized to a $[-1,1]$ scale. The inten sities of PET images were normalized with z-score normalization. We used the Medical Open Network for AI (MONAI) [14] software transformation packages to rescale and normalize the intensities of the PET/CT images. Image proces sing steps used in this manuscript are dis played in Figure 1A and 1B. 


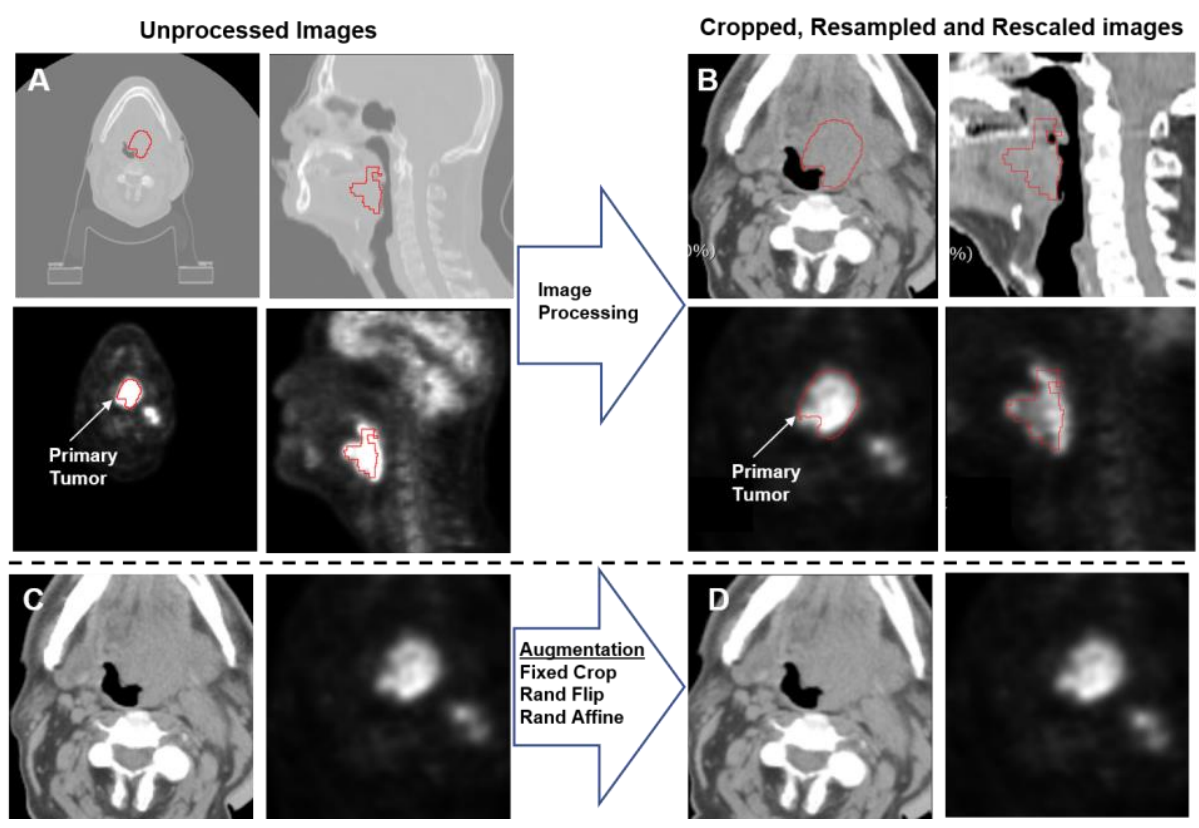

Fig. 1. An illustration of the workflow used for image processing. (A) Overlays of the provided ground truth tumor segmentation mask (used to highlight tumor location in the image but not used in any analysis done in the paper) and the original CT (top) and PET (bottom) images. (B) Illustration of data augment ation that included cropping, random flips, and affine transformations used during the model training.

\subsection{Clinical Data}

Clinical data for patients were provided in .csv files, which included PFS outcome information and clinical variables. As per the HECKTOR website, "the progression is defined based on RECIST criteria: either a size increase of known lesions (change of T and or N), or appearance of new lesions (change of $\mathrm{N}$ and/or M). Disease-specific death is als o considered a progression event for patients previously considered stable.". We used all the clinical variables provided by the HECKTOR Challenge with complete fields (no NaN values present) for all patients. These variables included Center ID, Gender, Age, TNM edition, chemotherapy status, TNM group, T-stage, N-stage, and $\mathrm{M}$-stage (9 variables in total). We elected not to use variables with incomplete fields (NaN values present), i.e., performance status, smoking status, etc., to avoid issues in the model building process. We mapped the individual T-, N-, and M- staging information to ordinal categorical variables in the following manner:T-stage: ('Tx': 0, 'T1': 1, 'T2': 2, 'T3': 3, 'T4': 4, 'T4a': 5, 'T4b': 6) - 7 classes; N-stage ('N0': 0, 'N1': 1, 'N2': 'N2a': 3, 'N2b': 4, 'N2c': 5, 'N3': 6) - 7 classes; M-stage ('Mx': 0, 'M0': 0, 'M1': 1) - 2 clas ses. We also mapped TNM group into an ordinal categorical variable based on information from the corres ponding TNM stage in the following manner: ('7 \& I': 0 , ' 8 \& I': 0, '7 \& II': 1, '8 \& II': 1, '7 \& III': 2, '8 \& III': 2, '7 \& IV': 3, '8 \& IV': 3, '7 \& IVA': 4, '8 \& IVA': 4, '7 \& IVB': 5, '8 \& IVB': 5, '7 \& IVC': 6, '8 \& IVC': 6) - 7 classes. Wethen 
medRxiv preprint doi: https://doi.org/10.1101/2021.10.14.21264955; this version posted October 17, 2021. The copyright holder for this preprint (which was not certified by peer review) is the author/funder, who has granted medRxiv a license to display the preprint in perpetuity.

It is made available under a CC-BY 4.0 International license .

used a min-max rescaling approach provided by the scikit-learn Python package [15] for data normalization. The s caler was in stantiated using only the training data and then applied to the test set, i.e., no data from the test set was allowed to leak into the training set. The normalized clinical data (9 variables perpatient) was then res haped such that each clinical variable is repeated $144 \mathrm{X} 144 \mathrm{X} 16$ times in the $\mathrm{x}, \mathrm{y}$, and $\mathrm{z}$ dimensions, respectively and therefore a volumetric image of size 144X144X144 was formed from the 9 concatenated images of each clinical variable. The volumetric image generated from the clinical data was used as a third channel with the CT and PET images to the DenseNet model(2.3).

\subsection{Model Architecture}

A deep learning convolutional neural network model based on the DenseNet121 architecture included in the MONAI s oftware package was used for the analysis. As shown in Figure 2, the network consists of 6, 12, 24, and 16 repetitions of dense blocks. Each dense block contained a pre-activation batch normalization, ReLU, and $3 \times 3 \times 3$ convolution followed by a batch normalization, ReLU, and $1 \times 1 x 1$ convolution (DenseNet-B architecture). The network implementation in PyTorch used was provided by MONAI [14]. The model has 3 in put channels with 144X144X144 each and 20 output channels representing different time intervals of the predicted survival probabilities (2.4).

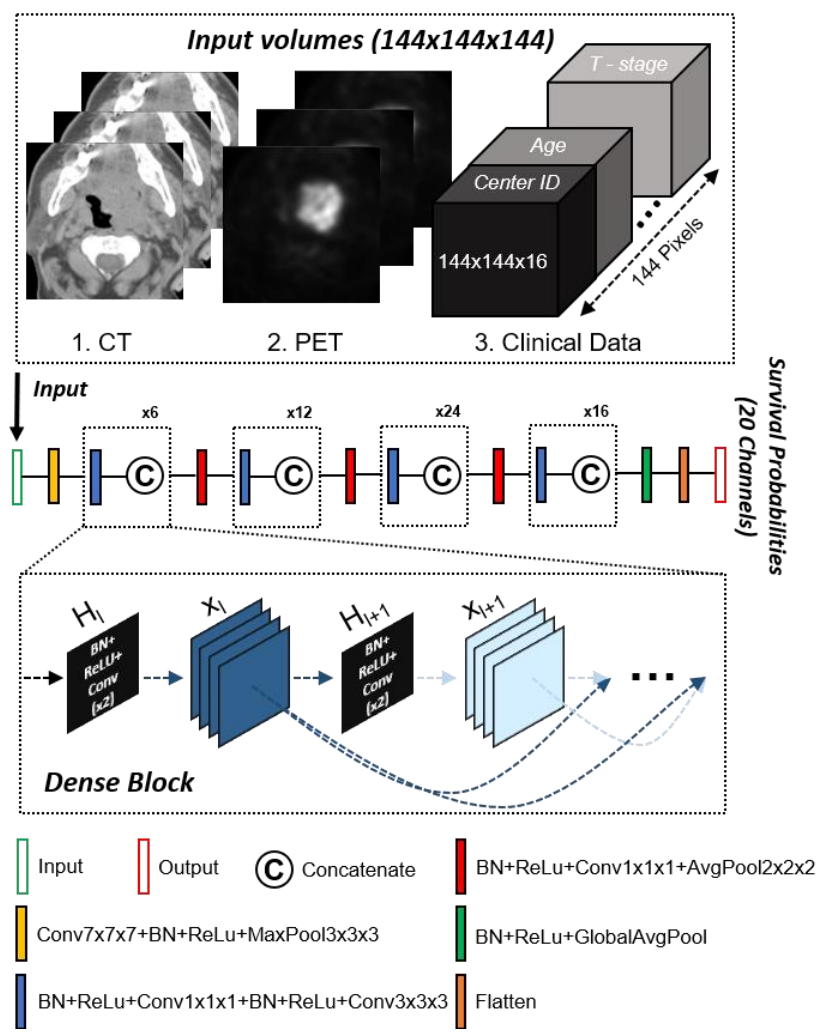


Fig. 2. Schematic of the Densenet 121 architecture used for the prediction model and the 3 input volumetric images representing CT, PET and clinical data. Clinical data is reformatted into a volumetric input for the model. The number of repeated dense blocks of $(6,12,24$, and 16) are given above each group of blocks.

\subsection{Model Implementation}

We used a 10 -fold cross-validation approach where the 224 patients fromthe training data were divided into 10 non-overlapping sets. Each set (22 patients) was used for model validation while the remaining 202 patients in the remaining sets were used for training, i.e., each set was used once for testing and 9 times for training. The processed PET and CT (2.1) were cropped to a fixed size of $(144,144,144)$ per image per patient. The clinical data was provided as a third channel to the model. We implemented additional data augmentation to the CT and PET channels, including random horizonal flips of 50\% and random affine transformations with an axial rotation range of 12 degrees and a scale range of $10 \%$. The image proces sing and data augmentation(2.1), network architecture (2.3) were used from the software packages provided by the MONAI framework [14]; code for these packages can be found at "https://github.com/ProjectMONAI/".

We implemented two main approaches for model building which utilizes imaging data only (Image), i.e., 2 channel input $-\mathrm{CT} / \mathrm{PET}$, or using imaging plus clinical data (Image+Clinical), i.e., 3 channel input $-\mathrm{CT} / \mathrm{PET}+$ clinical data. We used a batch size of 2 patients' images and clinical data and, therefore, the shape of the input tensor provided to the network (2.3) for the three-channel inputs was $(2,3,144,144,144)$ and for the two-channel inputs was $(2,2,144,144,144)$. The shape of the output tensor for 20 output channels was $(2,1,20)$ for both Image and Image+Clinical models. The model was trained for 800 iterations with learning rate of $\left(2 \times 10^{-4}\right.$ for iterations 0 to $300,1 \times 10^{-}$ ${ }^{4}$ for iterations 301 to 600 , and $5 \times 10^{-5}$ for iterations 601 to 800 ). We used Adamas the optimizer and a minus log-likelihood function as the loss function [16]. An implementation example of the log-likelihood function compatible with Keras was provided in (https://github.com/chestrad/survival_prediction/blob/master/loss.py) by Kim et al. [16]. We modified the code to match with PyTorch tensors used by MONAI [14]. In our implementation, we divided the total time interval of 9 years, which covers all values reported for PFS in the training dataset, into 20 discreateintervals of 164.25 days, representing the final 20 output channels of the network. The conditional probabilities of surviving in these intervals were obtained by applying a sig moid function on the 20 outputs channels of the network. As shown in the illustrative example of Fig. 3, all the time intervals preceding the events were set to 1 , and all other intervals were set to zero for both censored and uncensored patients (S vector). For uncensored patients (i.e., patients with censored status $\mathrm{p}=1$, where progression occurs), the time interval where progression occurs was set to 1 while all other intervals were set to $0(\bar{S}$ vector). The loss function is computed according to the equation shown in Fig.3.

We estimated the final PFS from the model predicted conditional probabilities by obtaining the summation of the cumulative probability of surviving each time interval times the duration of the time interval of 164.25 days. The cumulative probability of a time interval is obtained by multiplying the conditional probabilities of surviving all 
preceding time intervals times the time interval duration (i.e., PFS $^{\text {pred }}=$ $\sum_{k=1}^{20}\left(\prod_{i=1}^{k} S_{i}^{\text {pred }}\right) *($ time interval duration $=164.25)$

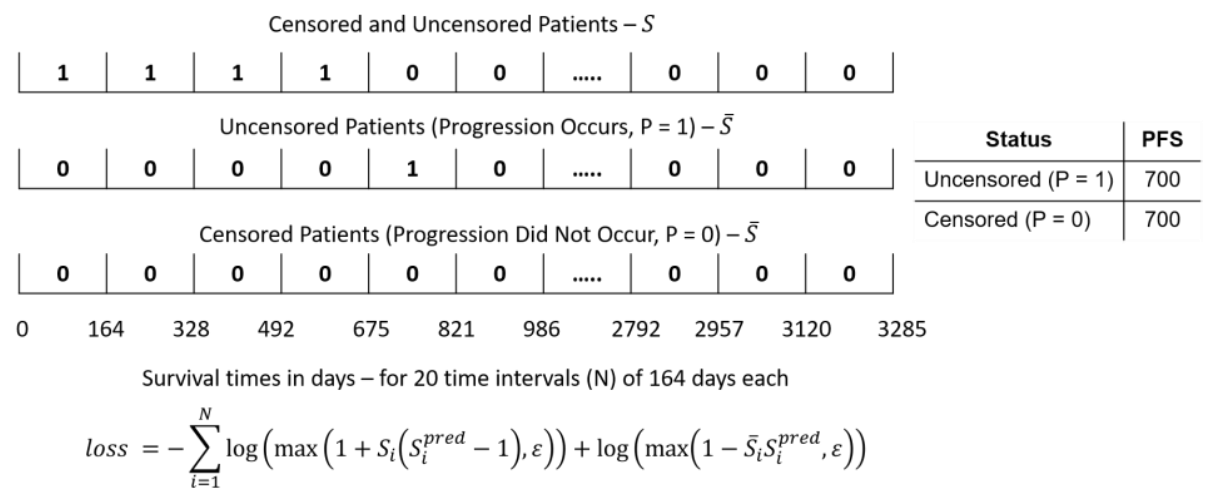

Fig. 3. A graphical illustration of the -log likelihood loss function used by the DenseNet model.

\subsection{Model Validation}

For each validation fold (i.e., 22 patients), we trained the DenseNet121 (2.3) on the remaining 202 patients. Therefore, we obtained 10 different trained models from $10-$ fold cros s-validation. We evaluated the performance of each separate model on the corresponding validation set using concordance index (C-index) function of the lifelines package (https://github.com/CamDavidsonPilon/lifelines) which accounts for both censored and uncensored data through the indication of the time events occurrence of progression (i.e., progression occurs - True or False). We estimated the mean C-index by averaging all the $\mathrm{C}$-indexvalues obtained fromeach fold. It is als o possible to measure the C-index by ignoring the events observed. Therefore, as an alternative metric, we als o meas ured this modified $\mathrm{C}$-indexin reporting results.

For the test set (101 patients), we implemented two different model ensembling approaches to estimate the PFS. In the first approach, we es timated the PFS for each patient by each model and then obtained the mean value of the 10 predicted PFS values (AVERAGE approach). In the second approach, we first estimated the mean conditional probability survival vector by getting the mean value for each time interval. Then we computed the cumulative survival probability for each interval to estimate the consensus PFS values from the 10 models (CONSENSUS approach).

\section{Results}

The performance (predicted PFS predictions vs. ground truth) of each s et of the 10-fold cross-validation procedure for the Image model and Image+Clinical model are shown in Figure 4 and Figure 5, respectively. For both models, most individual fold predictions were not significantly different from the ground truth PFS $(p>0.05)$, except sets 6 
medRxiv preprint doi: https://doi.org/10.1101/2021.10.14.21264955; this version posted October 17, 2021. The copyright holder for this preprint (which was not certified by peer review) is the author/funder, who has granted medRxiv a license to display the preprint in perpetuity.

It is made available under a CC-BY 4.0 International license .

and 8 for the Image model and sets 2 and 6 for the Image+Clinical model. The cumulative mean performance measured over all folds forboth models are shown in Table 1. Both models offered similar performance, regardless of the $\mathrm{C}$-index calculation method.
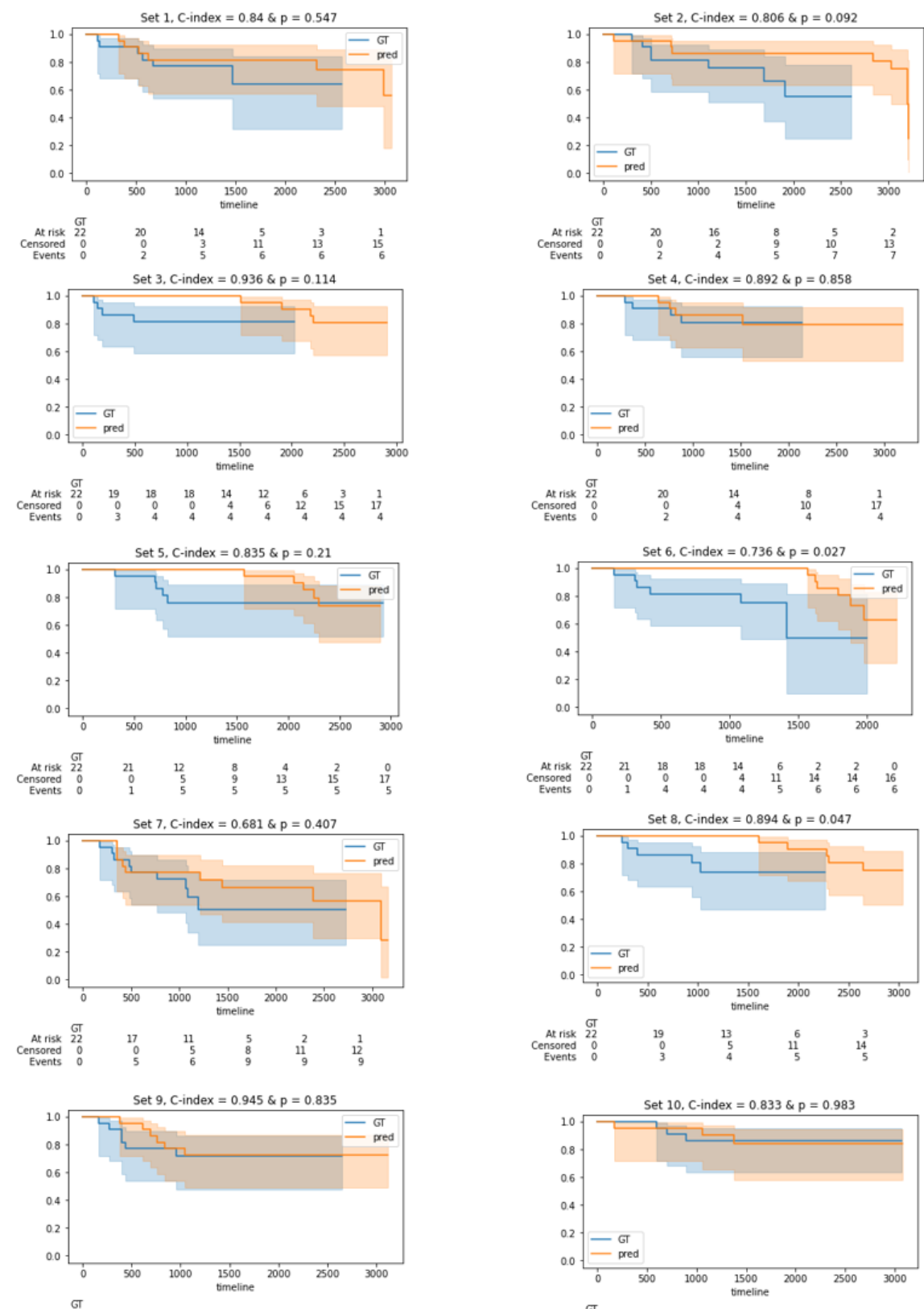

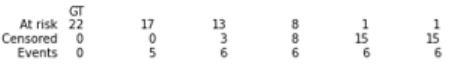
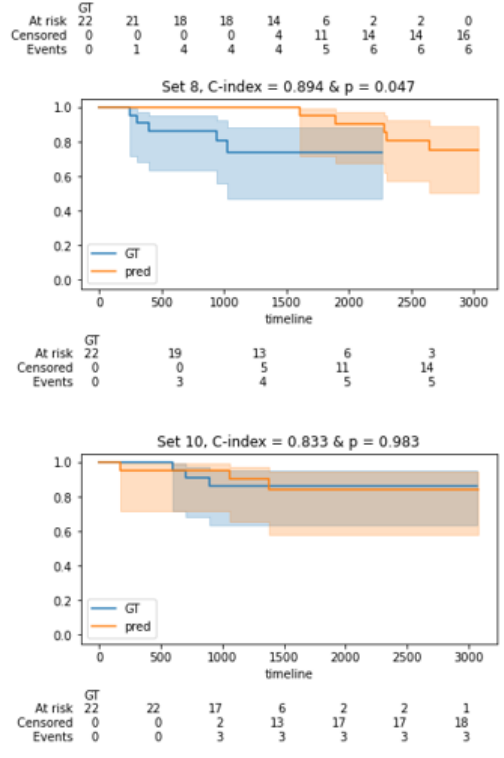
medRxiv preprint doi: https://doi.org/10.1101/2021.10.14.21264955; this version posted October 17, 2021. The copyright holder for this preprint (which was not certified by peer review) is the author/funder, who has granted medRxiv a license to display the preprint in perpetuity.

It is made available under a CC-BY 4.0 International license.

Fig. 4. Kaplan Meier plots showing survival probabilities as a function of time in days for the ground truth (GT) PFS and the predicted PFS by the model using only imaging data (i.e., CT and PET) for each validation set of 22 patients. The $\mathrm{C}$-index and the $\mathrm{p}$-value of the logrank test for the GT and predicted PFS are shown above each subplot.
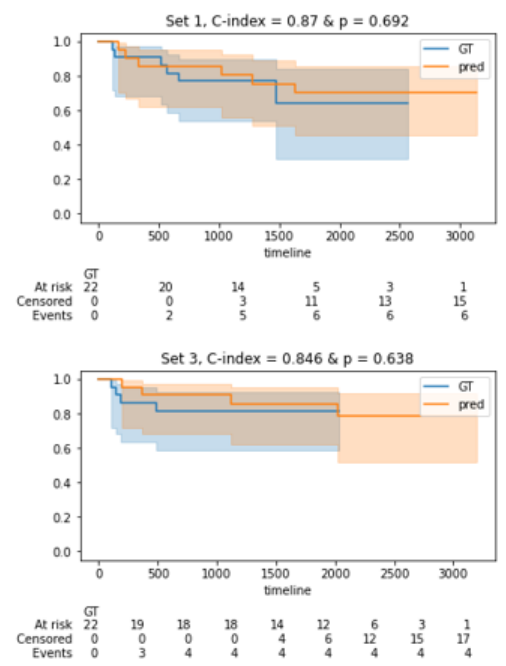

Set 5. C-index $=0.813 \& p=0.87$
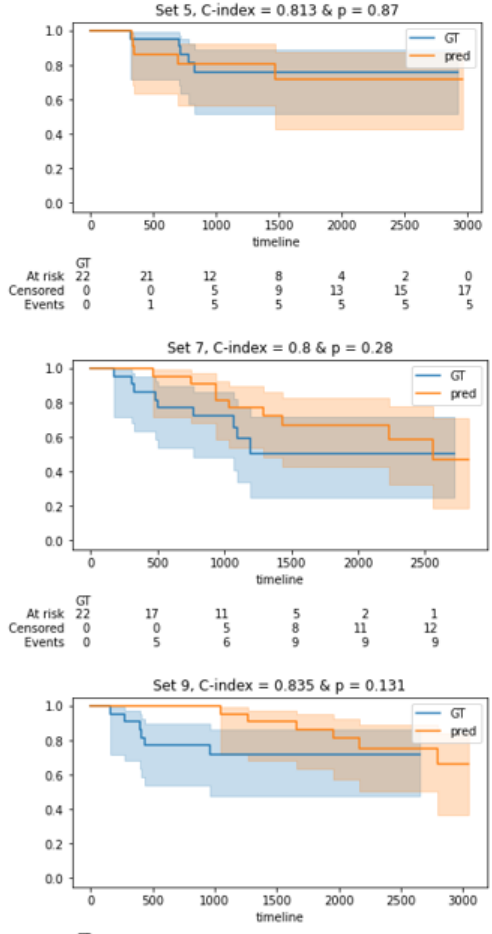

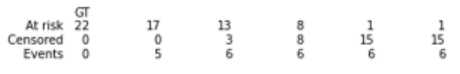
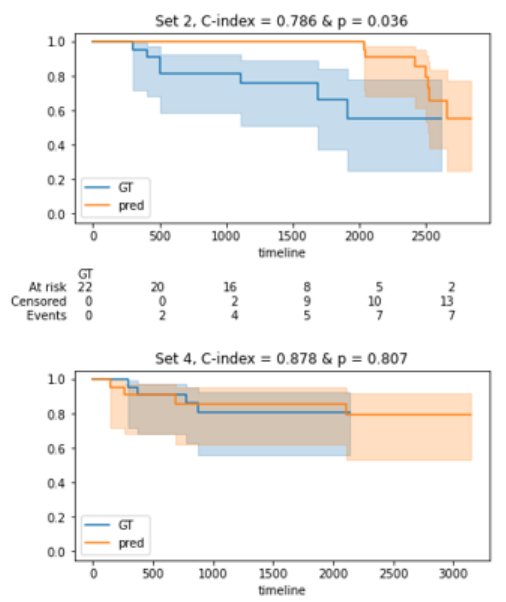

$\begin{array}{ccccc}\begin{array}{c}\text { At risk } \\ \text { GT }\end{array} & 20 & 14 & 8 & 1 \\ \text { Censored } \\ \text { Events }\end{array}$

Set 6. C-index $=0.791 \& p=0.007$

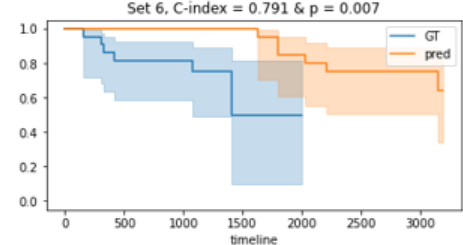

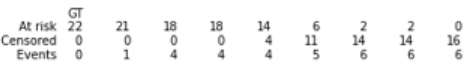
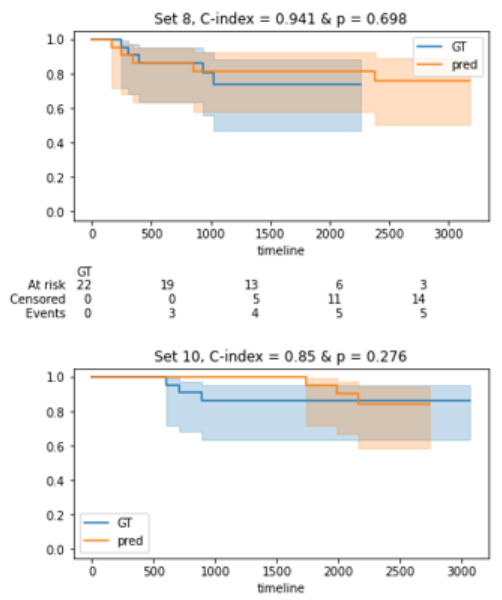

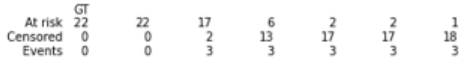


Fig. 5. Kaplan Meier plots showing survival probabilities as a function of time in days for the ground truth (GT) PFS and the predicted PFS by the model using both imaging and clinical data for each validation set of 22 patients. The $\mathrm{C}$-index and the $\mathrm{p}$-value of the logrank test for the GT and predicted PFS are shown above each subplot.

Table 1. Mean C-index across all cross-validation folds for evaluated models (Image, Image+Clinical). C-index can be calculated with and without observed events; both calculations are reported.

\begin{tabular}{lcc}
\hline Model & C-index (Events used) & C-index (no Events used) \\
\hline Image & $0.842 \pm 0.080$ & $0.620 \pm 0.082$ \\
Image + Clinical & $0.841 \pm 0.045$ & $0.622 \pm 0.067$ \\
\hline
\end{tabular}

When evaluated through external validation (testset), our model predictions fromeach cross-validation fold were ensembled using two methods (AVERAGE and CONSENSUS), leading to a total of four tested models (Image AVERAGE, Image CONSENSUS, Image+Clincial AVERAGE, Image+Clinical CONSENSUS). The results on the test set for these separate models are shown in Table 2. The highest performing model overall was the Image+Clinical model with a C-index of 0.694 .

Table 2. Progression-free survival prediction measured using the $\mathrm{C}$-index for ensemble models submitted to the 2021 HECKT OR Challenge evaluation portal. T wo ensemble approaches were tested(AVERAGE, CONSENSUS). * The exact method of C-index calculation was not provided but can be inferred to be with no events used.

\begin{tabular}{lc}
\hline Model & C-index* \\
\hline Image AVERAGE & 0.645 \\
Image + Clinical AVERAGE & 0.689 \\
Image CONSENSUS & 0.651 \\
Image + Clinical CONSENSUS & 0.694 \\
\hline
\end{tabular}

\section{Discussion}

In this study, we haveutilized deep learning approaches based on DenseNet applied to $\mathrm{PET} / \mathrm{CT}$ images and clinical data of HNSCC patients to predict PFS. The determination of prognostic outcomes is an unmet need for HNSCC patients that could improveclinical decision-making processes. While the performance of our models (as measured in C-index without events observed) is not ideal, they are reasonable within the context of prognostic prediction, which is known to be notoriously complex task [6]. Our main innovation stems from the use of ensembling approaches applied to predictions from internal cross-validation, which seems to reasonably improve overall performance on unseen data.

We evaluated two approaches (PET/CT inputs with and without clinical data) for internal validation through a 10 -fold cross-validation ap proach in the training set to gauge 
performance before applying the models to the test set. When investigating individual cross-validation sets, we find that performance for both models is often comparable to the ground truth PFS. Specifically, when utilizing C-index with observed events information considered, models could reach fairly high prediction performance, with values up to 0.842 for the Image model. Compared to PFS prediction models evaluated with the C-index in other studies, this is notably higher [5]. However, when implementing $\mathrm{C}$-index calculations without considering observed events information, this value drops precipitously for both models. Specifically, a maximum value of 0.622 is achieved, which is more consistent with previously reported values for similar tasks. Interestingly, the addition of clinical data generally did not make any noticeable improvements in model performance, regardless of the $\mathrm{C}$-index metric calculation method, possibly indicating the majority of informative data was contained within the PET/CT images. This observation runs counter to results seen in other clinical prediction models, where the addition of clinical data to imaging information often improves performance [6].

For the external validation (testing set) we further investigate the effect of specific model ensembling techniques through the AVERAGE and CONSENSUS methods. Generally, the CONSENSUS method (consensus from cumulative survival probability derived from mean conditional probability survival vectors) seems to offer per-formance gains over the A VERAGE method. Importantly, model performance on the test set is improved compared to the training set (assuming C-indexcalculation did notincorporate the observed events). Specifically, we achieve C-index gains of approximately 0.07 for test set performance. This may be due to the ensemble approach adding generalizability acquired from the combined inference capabilities shared across multiple cros s-validation sets. Interestingly, while adding clinical data did not make noticeable differences in the training set, this was not the case in the test set, which offered substantial C-indexgains of approximately 0.04 . The discrepancy may be explained by clinical data being more relevant when used in conjunction with model ensembling. Model ensembling is known to be a powerfultechnique in machine learning generally. This has been reiterated in other tasks for the HECKTOR Challenge, where ensembling provided impressive performance gains for tumor segmentation [17]. Therefore, we emphasize that model ensembling techniques may also be relevant for deep learning prediction models in HNSCC.

While we have taken steps to ensure a robust analysis, our study contains several limitations. Firstly, while we have included clinical datain our model-building process, we have not included all the data initially provided by the HECKTOR Challenge. Specifically, we have not inclu ded clinical data with incomplete fields (NaNs) for any patients, such as tobacco status, alcohol status, and human papillomavirus status. Importantly, it is precisely these variables that are often the most highly correlated to prognosis in HNSCC $[2,18]$. Therefore, data imputation techniques or related methods should be implemented in future studies to fully realize available clinical variables' discriminative capabilities. A second limitation of our approach is we have used an out-of-the-box DenseNet architecture that has not been specifically optimized for imaging data combined with tabular clinical data. Moreover, to ensure that we could utilize our DenseNet 
medRxiv preprint doi: https://doi.org/10.1101/2021.10.14.21264955; this version posted October 17, 2021. The copyright holder for this preprint (which was not certified by peer review) is the author/funder, who has granted medRxiv a license to display the preprint in perpetuity.

It is made available under a CC-BY 4.0 International license.

architecture with an additional channel input for clinical data, we have concatenated clinical data into a 3D volume for modelinput. While using an open-source commonly available approach improves reproducibility, further studies should determine how architectural modifications can be made to optimize performance on this specific task. Additionally, in our model design process, we have selected discrete intervals based on obs ervations in the training data. However, this assumes the training data fully encapsulates the PFS landscape, which may not necessarily be the case. Finally, since deep learning ens embling applied to PFS prediction is relatively understudied, we have approached modelen sembling of individual cross-validation folds through relatively simple methods that involve linear combinations of individual model predictions. More complex methods for deep learning ensembling [19] may provide additional predictive power for PFS tasks.

\section{Conclusion}

Us ing PET/CT and clinical data inputs, we developed, trained, and validated a series of deep learning models that could predict PFS in HNSCC patients in large-s cale datasets provided by the 2021 HECKTOR Challenge. Cross-validation performance on the training set achieved mean $\mathrm{C}$-index values of up to 0.622 ( 0.842 with alternative $\mathrm{C}$ index calculations). Simple model ens embling approaches improved this performance further with reported C-index values up to 0.694 on the testing set. While our models offer modest performance on test data, these methods can be enhanced through additional clinical in puts, improved architectural modifications, and alternative ensembling approaches.

Acknowledgements. M.A.N. is supported by a National Institutes of Health (NIH) Grant (R01 DE028290-01). K.A.W. is supported by a training fellowship from The University of Texas Health Science Center at Houston Center for Clinical and Translational Sciences TL1 Program(TL1TR003169), the American Legion Auxiliary Fellowship in Cancer Research, and a NIDCR F31 fellowship (1 F31 DE031502-01). C.D.F. received funding from the National Institute for Dental and Craniofacial Research Award (1R01DE025248-01/R56DE025248) and Academic-Industrial Partnership Award (R01 DE028290), the National Science Foundation(NSF), Divis ion of Mathematical Sciences, Joint NIH/NSF Initiative on Quantitative Approaches to Biomedical Big Data (QuBBD) Grant (NSF 1557679), the NIH Big Data to Knowledge (BD2K) Programof the National Cancer Institute (NCI) Early Stage Development of Technologies in Biomedical Computing, Informatics, and Big Data Science Award (1R01CA214825), the NCI Early Phase Clinical Trials in Imaging and Image-Guided Interventions Program(1R01CA218148), the NIH/NCI Cancer Center Support Grant (CCSG) Pilot Research Program Award from the UT MD Anderson CCSGRadiation Oncology and Cancer Imaging Program(P30CA016672), the NIH/NCI Head and Neck Specialized Programs of Res earch Excellence (SPORE) Developmental Research Program Award (P50 CA097007) and the National Ins titute of Biomedical Imaging and 
Bioengineering (NIBIB) Research Education Program (R25EB025787). He has received direct industry grant support, speaking honoraria and travel funding fromElekta AB.

\section{References}

1. Johnson, D.E., Burtness, B., Leemans, C.R., Lui, V.W.Y., Bauman, J.E., Grandis, J.R.: Head and neck squamous cell carcinoma. Nat. Rev. Dis. Prim. 6, 1-22 (2020).

2. Chow, L.Q.M.: Head and neck cancer. N. Engl. J. Med. 382, 60-72 (2020).

3. Budach, V., Tinhofer, I.: Novel prognostic clinical factors and biomarkers for out come prediction in head and neck cancer: a systematic review. Lancet Oncol. 20, e313-e326 (2019).

4. Goel, R., Moore, W., Sumer, B., Khan, S., Sher, D., Subramaniam, R.M.: Clinical practice in PET/CT for the management of head and neck squamous cell cancer. Am. J. Roent genol. 209, 289-303(2017).

5. Haider, S.P., Burtness, B., Yarbrough, W.G., Payabvash, S.: Applications of radiomics in precision diagnosis, prognostication and treatment planning of head and neck squamous cell carcinomas. Cancers head neck. 5, 1-19 (2020).

6. Chinnery, T., Arifin, A., Tay, K.Y., Leung, A., Nichols, A.C., Palma, D.A., Mattonen, S.A., Lang, P.: Utilizing Artificial Intelligence for Head and Neck Cancer Outcomes Prediction From Imaging. Can. Assoc. Radiol. J. 72, 73-85 (2021).

7. Hosny, A., Aerts, H.J., Mak, R.H.: Handcrafted versus deep learning radiomics for prediction of cancer therapy response. Lancet Digit. Heal. 1, e106-e107 (2019).

8. Sun, Q., Lin, X., Zhao, Y., Li, L., Yan, K., Liang, D., Sun, D., Li, Z.-C.: Deep learning vs. radiomics for predicting axillary lymph node metastasis of breast cancer using ultrasound images: don't forget the peritumoral region. Front. Oncol. 10, 53 (2020).

9. Avanzo, M., Wei, L., Stancanello, J., Vallières, M., Rao, A., Morin, O., Mattonen, S.A., El Naqa, I.: Machine and deep learning methods for radiomics. Med. Phys. 47, e185e202 (2020).

10. Hosny, A., Parmar, C., Coroller, T.P., Grossmann, P., Zeleznik, R., Kumar, A., Bussink, J., Gillies, R.J., Mak, R.H., Aerts, H.J.W.L.: Deep learning for lung cancer prognostication: a retrospective multi-cohort radiomics study. PLoSMed. 15, e1002711 (2018).

11. Willemink, M.J., Koszek, W.A., Hardell, C., Wu, J., Fleischmann, D., Harvey, H., Folio, L.R., Summers, R.M., Rubin, D.L., Lungren, M.P.: Preparing medical imaging data for machine learning. Radiology. 295, 4-15 (2020).

12. AIcrowd MICCAI 2020: HECKTOR Challenges.

13. Andrearczyk, V., Valentin, O., Mario, J., Vallières, M., Castelli, J., Elhalawani, H., Boughdad, S., Prior, J.O., Depeursinge, A.: Overview of the HECKTOR challenge at MICCAI 2020: Aut omatic Head and Neck Tumor Segmentation in PET/CT.

14. Project MONAI.

15. Pedregosa, F., Varoquaux, G., Gramfort, A., Michel, V., Thirion, B., Grisel, O., Blondel, M., Prettenhofer, P., Weiss, R., Dubourg, V.: Scikit-learn: Machine learning in Python. J. Mach. Learn. Res. 12, 2825-2830 (2011). 
medRxiv preprint doi: https://doi.org/10.1101/2021.10.14.21264955; this version posted October 17, 2021. The copyright holder for this preprint (which was not certified by peer review) is the author/funder, who has granted medRxiv a license to display the preprint in perpetuity.

It is made available under a CC-BY 4.0 International license .

16. Kim, H., Goo, J.M., Lee, K.H., Kim, Y.T., Park, C.M.: Preoperative CT -based Deep Learning Model for Predicting Disease-Free

Survival in Patients with Lung Adenocarcinomas. https://doi.org/10.1148/radiol.2020192764. 296, 216-224 (2020). https://doi.org/10.1148/RADIOL.2020192764.

17. Iantsen, A., Visvikis, D., Hatt, M.: Squeeze-and-excitation normalization for aut omated delineation of head and neck primary tumors in combined PET and CT images. In: 3D Head and Neck T umor Segmentation in PET/CT Challenge. pp. 37-43. Springer (2020).

18. Leemans, C.R., Snijders, P.J.F., Brakenhoff, R.H.: The molecular landscape of head and neck cancer. Nat. Rev. Cancer. 18, 269-282 (2018).

19. Ganaie, M.A., Hu, M.: Ensemble deep learning: A review. arXiv Prepr. arXiv2104.02395. (2021). 